\title{
CTNNB1 Mutation Analysis
}

National Cancer Institute

\section{Source}

National Cancer Institute. CTNNB1 Mutation Analysis. NCI Thesaurus. Code C157177.

A procedure used to detect and identify mutations in the CT NNB1 gene. 